\title{
Two Cases of Primary Extracutaneous Melanoma: Primary Gastric Melanoma and Primary Melanoma of the Lung
}

\author{
Alden Holmes* and Josiah Chung
}

Department of Hematology and Oncology, St. Bernardine's Hospital, 2101 N. Waterman Ave. San Bernardino, CA 92404, USA

*Corresponding author: Alden Holmes, Department of Hematology and Oncology, St. Bernardine's Hospital, 2101 N. Waterman Ave. San Bernardino, CA 92404, USA, Tel: (714) 394-2124; E-mail: gholm002@ucr.edu

Rec Date: August 28, 2017, Acc Date: September 14, 2017, Pub Date: September 16, 2017

Citation: Holmes A, Chung J (2017) Two Cases of Primary Extracutaneous Melanoma: Primary Gastric Melanoma and Primary Melanoma of the Lung. Med Case Rep Vol.3 No.4:39.

\section{Abstract}

Primary extracutaneous melanoma is an exceedingly rare occurrence, and primary extracutaneous melanomas arising in tissues thought to be devoid of melanocytes are even rarer. The paper that follows describes two such cases, one case of primary gastric melanoma and one case of primary melanoma of the lung. Both cases were treated solely with surgical excision of the lesion, and the patients have experience no recurrent disease. Novel approaches to treat extracutaneous melanomas are also discussed.

Keywords: Extracutaneous melanoma; Primary gastric melanoma; Primary melanoma of the lung; Vemurafenib; Ipilimumab

\section{Introduction}

In $2014,76,100$ individuals were diagnosed with melanoma and 9710 individuals died of melanoma in the United States [1], making melanoma the $5^{\text {th }}$ most common cause of cancer in United States men and the $6^{\text {th }}$ most common cause of death in United States women [2].

While melanoma is predominantly considered a cutaneous cancer, extracutaneous melanoma does occur. Bishop et al. identified the rate of extracutaneous melanoma to be $1 / 100,000$ in Western Countries [3], or approximately $1.4 \%$ of the total number of melanoma cases [2]. Extracutaneous melanomas have been identified in tissues known to harbor melanocytes: ocular, mucosal (including mucosal of the oral cavity, esophagus, and anorectal region), and leptomeningeal [2]. During development, melanocytes migrate from the neural crest along identifiable pathways. The function of extracutaneous melanocytes is not fully understood, with theories suggesting a potential antimicrobial or immunologic role [2].

Interestingly, primary melanomas have also been identified in visceral organs that lack melanocytes. Roughly 19 cases of primary gastric melanoma and 40 cases of primary pulmonary melanoma have been identified, for example $[4,5]$.

Several theories for the development of primary visceral melanoma exist. One theory includes aberrant neural crest cell migration during development $[2,6]$. Other theories explain the existence of primary visceral melanoma with metaplasia, for example amine precursor uptake and decarboxylation cells developing into primary gastrointestinal melanoma [6] and neuroendocrine system cells in the submucosal bronchial glands undergoing melanocytic differentiation to produce primary melanoma of the lung $[4,7]$. A third and final theory regarding the development of primary visceral melanoma is regression of a primary cutaneous melanoma explaining the lack of cutaneous findings in the documented cases of primary visceral melanoma [2].

Two case reports of primary visceral melanoma will follow. The first describes a case of primary gastric melanoma, and the second describes a case of primary melanoma of the lung. A discussion regarding diagnosis and treatment options will follow each case.

\section{Case Study 1}

The first patient is a 68-year-old male with a history of neurogenic bladder as well as colon cancer status post resection. The patient had been receiving cystoscopies on an annual basis for his neurogenic bladder. One such cystoscopy demonstrated a papillary bladder tumor. A CT scan of the abdomen and pelvis with and without contrast was performed to evaluate for metastasis. The CT scan revealed a $4.1 \mathrm{~cm}$ mass along the posterior wall of the cardia stomach without lymphadenopathy. The remainder of the scan was unremarkable.

His past medical history included hypertension, hyperlipidemia, major depressive disorder, neurogenic bladder, and history of colon cancer. These conditions were being treated medically with lisinopril, atorvastatin, wellbutrin, abilify, viibryd, and jalyn. The patient's surgical history consisted of a partial colon resection for colon cancer and transurethral incision of the prostate.

Gastroenterology was consulted and presumed that the 4.1 $\mathrm{cm}$ mass was a gastrointestinal stromal tumor, and a robotic 
assisted partial gastrectomy with control of splenic bleeding as well as transurethral resection of the bladder tumor was performed. The patient's vitals were stable on admission, physical examination was unremarkable, and admission labs were unremarkable except for mild hyponatremia at 130 $\mathrm{mmol} / \mathrm{L}$.

A histological evaluation was performed on the surgical specimen, which demonstrated a $7.0 \times 6.0 \times 4.5 \mathrm{~cm}$ pleomorphic malignant neoplasm showing features consistent with malignant melanoma. The majority of the involved cells were pleomorphic spindle cells, occasionally multinucleated, arranged into sheets and fascicles. Tumor necrosis was also prominent. Immuno-histochemical staining was significant for positive $\mathrm{S}-100$, melan-A, and $\mathrm{HMB}-45$ staining, tumor markers found in cutaneous melanomas [8]. The specimen was negative for CD34 and DOG-1, effectively eliminating the tumor's presumed identity as a gastrointestinal stromal tumor. The proximal and distal margins of the specimen were free of tumor. No lymph nodes were sent to pathology. Separate testing indicated that the patient's tumor was V600E negative.

The patient was referred to dermatology to identify potential primary cutaneous sources that could have metastasized to gastric mucosa. No such primary cutaneous lesions have been found. Biopsied lesions have been identified as keloid, solar lentigo, seborrheic keratosis, squamous cell carcinoma in-situ, and basal cell carcinoma, all separate and distinct entities from melanoma that originates from dysplastic nevi. A PET scan was suggested and declined by the patient secondary to financial considerations. Additionally, systemic adjuvant chemotherapy was offered but the patient declined. There was no sign of recurrence after four months.

\section{Discussion}

As previously stated, only 19 cases of primary gastric melanoma have been recorded in medical literature [4]. While melanocytes are present in the oral cavity and esophagus, the stomach, small intestines, and colon are devoid of melanocytes [9]. $30 \%$ to $40 \%$ of these cases presented as detectable masses [10] or symptoms including abdominal pain, weight loss, melena, anemia, and axillary lymphadenopathy [2]. Metastasis to the liver as well as regional lymph nodes from primary gastric melanoma is common [11]. Our patient presented entirely asymptomatically. The gastric tumor was identified incidentally after a CT abdomen and pelvis related to the discovery of a papillary bladder tumor. Lymph nodes were not sampled during the surgical removal of the gastric melanoma for our patient because the tumor was presumed to be a gastrointestinal stromal tumor.

The gastrointestinal tract is a common sight of cutaneous melanoma metastasis. $60 \%$ of patients who died of cutaneous melanoma had metastasis to the gastrointestinal tract on autopsy [12]. For this reason, diagnostic criteria for gastric melanoma have been proposed that preclude this possibility. One set of diagnostic criteria for primary gastric melanoma includes the following: 1) single lesion of melanoma in the stomach proven by pathology, 2) no concurrent lesions in other site of the body, 3) no history of melanoma, 4) diseasefree survival of at least 12 months after curative surgery $[13,14]$. Usual markers for primary gastric melanoma include S-100, melan A, HMB-45, and tyrosinase [9].

Our patient complies with the proposed diagnostic criteria thusfar. The pathology sample exhibited characteristic melanoma findings, dermatological evaluation has yet to reveal any dysplasia related to melanoma, and the patient has no history of melanoma. This patient has also survived 10 months after surgery, nearing the 12 month disease-free survival criterion.

No universally recognized staging protocol exists for the majority of extracutaneous melanomas, making it difficult to compare the efficacy of treatment modalities in a meaningful manner. Extracutaneous melanomas are typically staged using systems for more common malignancies at various tumor sites [2]. The exception to this rule is mucosal melanoma of the head and neck for which tumor-node-metastasis (TNM) staging system was adopted in the seventh edition of The American Committee on Cancer (AJCC) cancer staging manual [2].

Treatment recommendations for extracutaneous melanomas are limited by the rarity of the condition. Common practice has been surgery with or without the addition of adjuvant radiotherapy and chemotherapy depending on practitioner judgment [2]. A case report by Wang et al. presented a primary esophago-gastric melanoma treated solely with debulking surgery that ended with the patient's death from diffuse metastatic disease two months after surgery. The extremely short survival led the research group to strongly recommend post-operative chemotherapy or radiotherapy [4]. This recommendation is not the norm, and the median survival of patients with primary gastric melanoma treated with surgery with or without adjuvant therapy based on physician discretion has been 5 months [15]. Clearly, additional treatment modalities are required.

Novel agents for the treatment of cutaneous melanoma are starting to be applied for the treatment of primary visceral melanomas. Bolzacchini et al. described managing a primary gastric melanoma with vemurafenib and ipilimumab. Metastasis to the liver and regional lymph nodes was present. Screening demonstrated that the tumor was V600E positive, indicating that vemurafenib was an appropriate therapy. The patient was treated with vemurafenib for six months. At this time, treatment was changed to ipilimumab secondary to tumor progression. Four doses of ipilimumab were administered until the patient passed away 11 months after tumor discovery [11]. This patient's survival was significantly longer than the 5 month median survival time expected for primary gastric melanoma and is an accomplishment when the extensive disease at discovery is considered. This treatment option would have been appropriate for our patient given his negative $\mathrm{V} 600 \mathrm{E}$ status, but the success of the treatment demonstrates that screening for V600 mutations should be standard of care for extracutaneous melanomas and that vemurafenib paired with ipilimumab should be considered a future possibility for the treatment of extracutaneous 
melanomas. Additional research is required to quantify the exact efficacy of these agents.

Another novel treatment being selectively applied to extracutaneous melanomas is KIT-inhibitors. Beading et al. found KIT mutation in $15.6 \%$ of extracutaneous melanomas tested [16], indicating that these tumors could be treated with KIT-inhibitors. Hodi et al. described a patient with metastatic rectal melanoma that was KIT positive. Surgical resection of the primary tumor was performed, and the patient was treated with imatinib as adjuvant therapy. The patient experienced a marked reduction in both the size and number of metastatic lesions over a four month treatment period [17] Minor et al. observed a complete remission for 15 months and two partial remissions lasting 1 month and 7 months when using sunitinib to treat mucosal melanomas [18]. No KIT testing was performed on our case report patient, but again, the promise of these therapies indicates that KIT testing should be standard of care for the management of extracutaneous melanomas.

Broader therapies are also being researched that have the potential to aid treatment of patients with extracutaneous melanoma who prove to be V600 and KIT negative. Sakamoto et al. described management of a patient with primary malignant melanoma of the esophagus. The mean survival with this diagnosis was 9.8 months. This particular patient had several distressing features, including lymph node and lung metastasis. The patient was treated with local INF- $\beta$ injections and conventional systemic chemotherapy. The patient was in partial remission 18 months after the conclusion of treatment. This finding lead Sakamoto et al. to recommend the application of INF- $\beta$ for palliative treatment of primary malignant melanoma of the esophagus [19]. Additional studies are required to determine if this treatment has broader applicability in the treatment of extracutaneous melanomas.

Similarly, Asakage et al. applied dendritic cell therapy to treat primary malignant melanoma of the esophagus. The patient had an ulcerating primary lesion without metastasis. The treatment team administered four intradermal injections of dendritic cells. The patient was adequate treated without any indication of relapse 2.5 years after the conclusion of treatment, handily surpassing the 9.8 month mean survival time [20]. Again, additional studies are required to determine if this treatment has broader applicability in the treatment of extracutaneous melanomas. However, the mechanism of action for both INF- $\beta$ and dendritic cells rely on stimulating the patient's immune system $[19,20]$. This suggests that these therapies could be applied in a broader context with similar results.

\section{Case Study 2}

The second patient is a 43 year old male with history of hypertension and hypothyroidism who presented to the emergency department with cough and fatigue for one week as well as bright red hemoptysis for four hours. The hemoptysis started on the day the patient was returning from a fishing trip in Mexico. The patient denied a history of pneumonia, tuberculosis, asthma, or lung problems as well as a family history of malignancy. The patient did not smoke tobacco, drink alcohol, or use illicit drugs.

The patient's vitals were significant for tachycardia with a pulse of 122 and an elevated blood pressure at 153/97. The patient's complete blood count revealed a mildly white blood count at $11.3 \times 103$ per $\mathrm{mcL}$ and normal hemoglobin at 12.9 $\mathrm{g} / \mathrm{dL}$. The patient's complete metabolic panel, coagulation studies, and liver enzymes were unremarkable. The only contributory findings on physical examination were coarse lung wheezes bilaterally and tachycardia. A CT angiogram of the chest with and without contrast was ordered, revealing a $7.6 \mathrm{~cm}$ ovoid left upper lobe mass concerning for malignancy as well as scattered diffuse nodular ground glass appearance likely secondary to infection. No other anomalies, including perihilar and mediastinal lymphadenopathy, were noted.

The emergency department physicians placed the patient on levaquin for a possible pneumonia, placed orders for PPD and quantiferon tests, as well as admitted the patient to an isolation room until the possibility tuberculosis was eliminated. A bronchoscopy and CT needled guided biopsy of the left upper lobe performed within days of admission showed inflammation with intra-alveolar hemosiderin laden macrophages and minute bits of necrotic tissue, but no evidence of malignancy. Immunohistochemistry was negative for HMB-45, melan-A and other routine stains. The preliminary pathology diagnosis was scar tissue.

Pulmonary function tests revealed that the patient was a candidate for pneumonectomy, and bronchoscopy and left upper lobe lobectomy via a left posteriolateral thoracotomy was performed. The $560 \mathrm{~g}, 18.2 \times 13.5 \times 5.5 \mathrm{~cm}$ sample contained a large $9.0 \times 8.5 \times 5.5 \mathrm{~cm}$ cavitating, hemorrhagic mass. Two lymph nodes were submitted with the surgical specimen. The patient received two units of packed red blood cells for post-operative hemoglobin of $7.5 \mathrm{~g} / \mathrm{dL}$. No other complications were noted.

Pathology described as a spindle cell neoplasm demarcated from the adjacent lung parenchyma by a pseudocapsule. The tumor cells contained ovoid, open nuclei with prominent nucleoli. Occasional pleomorphic forms as well as pigmented cytoplasm were identified. Surgical margins were clear. Neither the left upper lobe lymph node nor the left intralobular lymph node submitted with the specimen contained metastatic melanoma. Immunohistochemical staining was positive for S-100, melan-A, and HMB-45 and diagnosed the lesion as a malignant melanoma. Subsequent V600E testing was negative. Dermatological evaluation as well as positron emission tomography is pending.

\section{Discussion}

Similar to primary gastric melanoma, primary melanoma of the lung is rare due to the absence of melanocytes in that tissue. Only 40 cases of primary melanoma of the lung have been identified in medical literature since 19165. The average age of diagnosis was 54, with ages ranging from 29-902. Presenting symptoms included cough, hemoptysis, 
postobstructive pneumonia, lobar collapse, and atelectasis [2] Our patient presented with hemoptysis as his chief complaint and was diagnosed with pneumonia in the emergency department.

Primary melanoma of the lung is initially viewed with suspicion secondary to the tendency of melanoma to metastasize to the lung. For this reason, the Armed Forced Institute of Pathology suggest the following criteria for the diagnosis of primary melanoma of the lung: 1) presence of a solitary lesion, 2) malignant melanoma confirmed by immunohistochemistry or electron microscopy, 3) no past history of excision or fulgration of a cutaneous, mucous membrane, or ocular lesion, 4) central pulmonary lesion, and 5) no demonstrable tumor elsewhere at the time of diagnosis [21].

Our patient had a single cavitating pulmonary lesion that exhibited positive S-100, melan-A, and HMB-45 staining and lacked any history of cutaneous or mucosal melanoma, meeting the majority of the diagnostic criteria. He has not yet had a positron emission tomography scan or a dermatological evaluation to conclusively rule out the possibility to tumor elsewhere.

\section{Conclusion and Recommendations}

Treatment recommendations for primary melanoma of the lung are similar to the recommendations for primary gastric melanoma. Common practice has been surgery, including complete lymph node resection, with or without the addition of adjuvant radiotherapy and chemotherapy based on practitioner judgment [2]. $65 \%$ of patients with primary melanoma of the lung survived less than 18 months following these treatment recommendations [5]. As with primary gastric melanomas, all mucosal melanomas should be screened for V600 and KIT mutations to determine the applicability of novel therapies that target these mutations. Interferon injections and dendritic cell therapy are possible adjuvant therapies that can be administered when tumors are V600 and KIT negative. Given the rarity of primary mucosal melanomas, the path to strong treatment recommendations remains unclear.

\section{References}

1. Avilés-izquierdo JA, Molina-lópez I, Rodríguez-lomba $E$, Marquez-rodas I, Suarez-fernandez R, et al. (2016) Who detects melanoma? Impact of detection patterns on characteristics and prognosis of patients with melanoma. J Am Acad Dermatol 75: 967-974.

2. Mihajlovic M, Vlajkovic S, Jovanovic P, Stefanovic V (2012) Primary mucosal melanomas: A comprehensive review. Int J Clin Exp Pathol 5: 739-753.

3. Bishop KD, Olszewski AJ (2014) Epidemiology and survival outcomes of ocular and mucosal melanomas: A populationbased analysis. Int J Cancer 134: 2961-2971.

4. Wang L, Zong L, Nakazato H, Wang WY, Li CF, et al. (2016) Primary advanced esophago-gastric melanoma: A rare case. World J Gastroenterol 22: 3296-301.
Kyriakopoulos C, Zarkavelis G, Andrianopoulou A (2017) Primary pulmonary malignant melanoma: Report of an important entity and literature review. Case Rep Oncol Med 2017:8654326.

6. Li H, Fan Q, Wang Z, Xu H, Li X, et al. (2012) Primary malignant melanoma of the duodenum without visible melanin pigment: $A$ mimicker of lymphoma or carcinoma. Diagn Pathol 7: 74.

7. Wilson RW, Moran CA (1997) Primary melanoma of the lung: A clinicopathologic and immunohistochemical study of eight cases. Am J Surg Pathol 21: 1196-1202.

8. Sanders GB (1998) Comparison of immunohistochemical staining of the novel antibody melan-A with S100 protein and HMB-45 in malignant melanoma and melanoma variants. Histopathology 32: 139-146.

9. Song W, Liu F, Wang S, Shi H, He W, et al. (2014) Primary gastric malignant melanoma: Challenge in preoperative diagnosis. Int J Clin Exp Pathol 7: 6826-6831.

10. Sabanathan S, Eng J, Pradhan GN (1989) Primary malignant melanoma of the esophagus. Am J Gastroenterol 84: 1475-1481.

11. Bolzacchini E, Marcon I, Bernasconi G, Pinotti G (2016) Primary melanoma of the stomach treated by BRAF inhibitor and immunotherapy. Dig Liver Dis 48: 974.

12. Patel JK, Didolkar MS, Pickren JW, Moore RH (1978) Metastatic pattern of malignant melanoma. A study of 216 autopsy cases. Am J Surg 135: 807-810.

13. Chang AE, Karnell LH, Menck HR (1998) The National Cancer Data Base report on cutaneous and non-cutaneous melanoma: A summary of 84,836 cases from the past decade. The American College of Surgeons Commission on Cancer and the American Cancer Society. Cancer 83: 1664-1678.

14. Elsayed AM, Albahra M, Nzeako UC, Sobin LH (1996) Malignant melanomas in the small intestine: A study of 103 patients. Am J Gastroenterol 91: 1001-1006.

15. Cheung MC, Perez EA, Molina MA, Jin X, Gutierrez JC, et al. (2008) Denying the role of surgery for primary gastrointestinal tract melanoma. J Gastrointest Surg 12: 731-738.

16. Beadling C, Jacobson-Dunlop E, Hodi FS, Le C, Warrick A, et al. (2008) KIT gene mutations and copy number in melanoma subtypes. Clin Cancer Res 14: 6821-6828.

17. Hodi FS, Friedlander P, Corless CL, Heinrich MC, Mac-Rae S, et al. (2008) Major response to imatinib mesylate in KIT-mutated melanoma. J Clin Oncol 26: 2046-51.

18. Minor DR, Kashani-Sabet M, Garrido M, O'Day SJ, Hamid O, et al. (2012) Sunitinib therapy for melanoma patients with KIT mutations. Clin Cancer Res 18: 1457-63.

19. Sakamoto $H$, Uedo $N$, lishi $H$, Higashino $K$, Ishihara $R$, et al. (2003) Treatment of primary malignant melanoma of the esophagus with endoscopic injection of interferon-beta combined with systemic chemotherapy: A case report. Gastrointest Endosc 57: 773-777.

20. Asakage M, Kitayama J, Tsuno NH, Komuro $\mathrm{Y}$, Kaisaki S, et al. (2005) Primary malignant melanoma of the esophagus treated by esophagectomy and adjuvant dendritic-cell therapy. J Gastroenterol 40: 545-546.

21. Mahowald MK, Aswad BI, Okereke IC, Ng T (2015) Long-term survival after pneumonectomy for primary pulmonary malignant melanoma. Ann Thorac Surg 99: 1428-1430. 